\title{
Pasarelas exóticas entre Latinoamérica y EE.UU.: Braniff Airways y "El fin del aeroplano plano"
}

\author{
Exotic Runways Spanning the US and Latin America: Braniff Airways and "The \\ End of the Plain Plane"
}

\author{
Phil Tiemeyer \\ tiemeyerp@ksu.edu \\ Kansas State University, Estados Unidos
}

Cita sugerida: Tiemeyer, P. (2021). Pasarelas exóticas entre Latinoamérica y EE.UU.: Braniff Airways y "El fin del aeroplano plano". Orbis Tertius, 26(33), e200. https://doi.org/10.24215/18517811e200
Resumen: En 1965, gracias a la adquisición de una nueva flota de jets, Braniff Airways - una aerolínea mediana con sede en Texas- anhelaba estar a la altura de sus competidores y conseguir así mejorar su participación de mercado, en especial, en las rutas internacionales que unían a los EE. UU. con varias ciudades de México y América del Sur. Este artículo examina cómo la apremiante situación financiera generada por esta millonaria adquisición daría lugar a una audaz campaña publicitaria y de mercadeo que finalmente sería responsable de la estilizada reinterpretación del viaje aéreo, que abandonaría los decorados serios pero sofisticados, y se adentraría en la nueva, colorida, glamorosa y ultramoderna "era del jet". Dicha transformación estilística implicó el trabajo conjunto entre tres grandes creativos de la década de 1960: la publicista Mary Wells, el diseñador de modas Emilio Pucci y el diseñador de interiores Alexander Girard. Tanto Pucci como Girard serían particularmente vitales en la creación de la colorida, pero sofisticada nueva imagen de Braniff, que definiría el carácter único y vanguardista de la "modernidad" en la era del jet. Asimismo, de forma intencionada estos diseñadores atribuirían su estética a la acogida del "primitivismo", en particular, empleando llamativas inspiraciones geométricas extraídas del arte de la América precolombina y otras civilizaciones del sur global.

Palabras clave: Braniff Airways, Era del jet, Sur Global, Transformación estilística, Arte precolombino.

Abstract: The mid-sized Texas-based airline Braniff Airways
aspired in 1965 to catch up to its competition by purchasing
a new fleet of jet airplanes and thereby improve its market
share, including on its international routes that linked the US
with various cities in Mexico and South America. This article
examines how the economic pressure of this substantial purchase
led to a bold marketing campaign that ultimately re-styled air
travel in the Jet Age from staid-but-sophisticated to colorful
and glamorously trendy. This stylistic transformation involved
a collaboration between three creative giants of the 1960 s:
advertising executive Mary Wells, fashion designer Emilio Pucci,
and interior designer Alexander Girard. Both Pucci and Girard
were particularly vital in creating a colorful, yet stylish image
for Braniff that rendered a uniquely up-to-date sense of what
Jet Age "modernity" would look like. At the same time, these 
designers consciously attributed their aesthetics to an embrace of "primitivism," especially to the bright, geometric inspirations they found in pre-modern art from Latin American civilizations and others across the Global South.

Keywords: Braniff Airways, Jet Age, Global South, Stylistic Transformation, Pre-Colombian Art.

\section{INTRODUCCIÓN: LA LLEGADA DEL JET ESTADOUNIDENSE AL "PAÍS DE LAS LLAMAS" - LOS CIELOS SOBRE LIMA Y ACAPULCO}

En ${ }^{1} 1958$, el legendario cantante estadounidense Frank Sinatra presentó su rítmica balada jazz, Come Fly With $M e$. Seductor, Sinatra lanza una invitación a su musa: "Come fly with me, we'll fly, we'll fly away" (Ven a volar conmigo, volaremos, volaremos lejos). Seguidamente, la canción se sucede en un collage aéreo de escenas y aventuras elitistas. Tras despegar en su gira por el mundo, "some exotic booze... in far Bombay" (degustando algún trago exótico... en el lejano Bombay), Sinatra se aproxima a casa. Sobrevuela América Latina, destino predilecto de los estadounidenses para los viajes de placer y ocio -la historia así lo demuestra-, que contenía todos los ingredientes para la escapada perfecta: en parte lujoso, en parte exótico y primitivo, siempre soleado y glamoroso. "Come fly with me" (Ven a volar conmigo), prosigue el cantante, "we'll float down to Peru. / In llama-land there's a one-man band / And he'll toot his flute for you" (flotaremos hasta Perú. / En la tierra de las llamas hay un hombre-orquesta / Y él tocará su flauta para ti). Más adelante, Sinatra nos sitúa en otro destino turístico, por entonces emergente y de creciente interés para la jet set de la época: el resort mexicano de Acapulco. "Just say those words and we'll beat the birds / Down to Acapulco Bay" (Solo di esas palabras y venceremos a los pájaros / Hasta la bahía de Acapulco), exclama su voz in crescendo, como si fuera ella misma el jet ${ }^{2}$ que despega hacia este paraíso tropical. "iEs perfecto para una luna de miel en las alturas, dicen!".

En octubre de ese mismo año, la compañía estadounidense Pan American Airways realiza su primer vuelo comercial de pasajeros desde Nueva York a Europa a bordo de un Boeing 707; por su parte, la futura British Airways (por entonces, BOAC) establecía también, con sus De Havilland Comets, un servicio regular de jet en sus rutas transatlánticas. ${ }^{3}$ El homenaje de Sinatra a algunos de los destinos más chic en América Latina fue, entonces, también premonitorio, e irradiaba la certeza de que "la era del jet" forjaría un camino incluso hacia los paraísos turísticos preferidos por los estadounidenses al sur de la frontera nacional. Tal como las rutas de Pan Am, que llegaban ahora a destinos tan al sur como Lima y Buenos Aires, sirvieron de puente para las actividades comerciales de los EE.UU. en América Latina en 1930, Come Fly With Me auguraba dos nuevas oleadas de estadounidenses listos para la conquista económica: primero, el llamado jet set -la renovada élite cosmopolita, compuesta por hombres de negocios y sus esposas, estrellas de la industria del entretenimiento como Sinatra, y los últimos aristócratas "de riqueza heredada" del Atlántico Norte-, cuyo estilo de vida nómada y opulento se aceleró mucho más gracias a la llegada del motor de reacción (Stadiem, 2014). Sin lugar a duda, serían estas élites las que ocuparían la sección de primera clase en los nuevos jets que pronto descenderían desde los cielos sobre Lima y Acapulco.

Pero, dado el inmenso tamaño de los aviones y su elevadísimo precio, los jet setters inevitablemente tendrían que ir acompañados de una segunda ola turística: un séquito de seguidores, la clase turista, cuyo considerable volumen sería decisivo en las inversiones de las aerolíneas en nuevos aviones de reacción, haciendo rentable su despliegue de rutas latinoamericanas. Por consiguiente, en las décadas de 1950 y 1960, el desafío que la era del jet imponía para Braniff Airways -aerolínea con sede en Texas-, y que sería la única otra compañía estadounidense con certificado para operar las rutas entre EE.UU. y América Latina junto a la célebre Pan Am, era traducir para estos dos públicos -la élite y la creciente clase media estadounidense-, la romántica invitación de Sinatra a acompañarlo en unas vacaciones en América Latina. ¿Podría esta aerolínea 
seducir a sus clientes tanto de primera clase como de clase turista con la promesa de que sus aventuras por Latinoamérica - una atractiva combinación de días soleados, lujo y exotismo-, ofrecería, simultáneamente, los encantos de la modernidad y el cautivador encuentro con lo primitivo?

Lo que sigue es, pues, el recuento económico y social de los esfuerzos de Braniff Airways por reconciliar las fuerzas, aparentemente antagónicas, entre lo "moderno" y lo "primitivo"; todo, con el propósito de ofrecer a los consumidores estadounidenses un servicio de jet a Latinoamérica que fuera lujoso a la par que asequible, exclusivo a la par que democrático, al menos lo suficiente como para estar al alcance de las masas. Fue así como, en 1965, Braniff reunió un equipo de analistas económicos, expertos en relaciones públicas y diseñadores de renombre mundial para forjar tal producto. El resultado, lanzado a bombo y platillo ese mismo año, anunciaría "El fin del aeroplano plano" con un producto que sería atractivo para las grandes masas de turistas estratificados de la era del jet, y que a su vez ayudaría a dar forma al turismo latinoamericano en los años venideros. Ciertamente, la campaña permitió a Braniff hacerse con una mayor cuota del mercado frente a Pan Am, su principal competidor, a la par que sirvió para contener amenazas de menor tamaño, como Varig, de Brasil, y Avianca, de Colombia, aerolíneas latinoamericanas que, a su vez, ponían su vista sobre los mercados turísticos estadounidenses como conquistas cruciales en la expansión de sus operaciones más al norte. El producto final ofrecido por Braniff en los comerciales de televisión y los relucientes anuncios de revistas era una experiencia a bordo más elegante y moderna, que a su vez haría realidad la llegada de más turistas estadounidenses a la "tierra de las llamas" y el éxito en cifras de los paquetes turísticos para quienes buscaban una "luna de miel perfecta a bordo" con destino a Acapulco.

El resto de este artículo examina, en primer lugar, los factores económicos que influyeron en el cambio de imagen de Braniff en la era del jet, y que permitieron a la empresa pagar la enorme deuda asumida por la compra de sus nuevos aviones. Después, el artículo revisa, de forma más específica, los diseñadores seleccionados para liderar esta transformación: el diseñador de modas Emilio Pucci, y el diseñador de interiores Alexander Girard, quienes invocaron tropos primitivistas, entre ellos los tonos vivos y las formas simplistas aprendidas del arte precolombino exhibido por las civilizaciones azteca, inca y maya (también, en el caso de Pucci, por las sociedades premodernas del sudeste asiático y de África). El resultado: un producto ultramoderno propio de la era del jet, no obstante, explotó el supuesto primitivismo latinoamericano para hechizar a un número cada vez mayor de turistas estadounidenses que desde ahora podrían hacerse con sus encantos.

\section{Economías DEL ESTILO EN LA "ERA DEL JET": "LOS TONOS VIBRANTES" De MÉXico Y SUDAMÉRICA}

Para Braniff Airways - por entonces una aerolínea mediana con sede en Dallas, Texas, centro urbano intermedio en crecimiento-, 1965 fue un año trascendental. Desde su primer vuelo en 1928, Braniff subsistió como aerolínea regional en el estrictamente regulado mercado del transporte aéreo de los Estados Unidos, gracias a la concesión de rutas en Texas, con destino al este, en Nueva Orleans, y al norte, en Kansas City. Los años posteriores a la Segunda Guerra Mundial permitieron a Braniff expandirse más allá del centro de los Estados Unidos. En 1952, una fusión a nivel doméstico permitió la expansión de la red de rutas a Chicago; años después, la compañía realizaría sus primeros vuelos entre Dallas y la costa este. Sin embargo, y lo que es más importante, Braniff finalmente se convertiría en una aerolínea internacional en 1948. Gracias a la concesión de rutas a América Latina por parte del gobierno estadounidense, Braniff sería, después de Pan Am - primer competidor-, la segunda compañía aérea de Estados Unidos con rutas a México, América Central y América del Sur (Cass, 2015).

Aun así, Braniff tenía muy poca visibilidad de marca en las principales ciudades de los Estados Unidos y en las metrópolis latinoamericanas. Por ejemplo, a mediados de los sesenta, solo un tres por ciento de los clientes potenciales en el área de Nueva York habían oído hablar de la aerolínea, lo que suponía una desventaja 
significativa en el aún más grande mercado de consumidores de los Estados Unidos (Harding Lawrence Markets an Airline, 1968). Además, Braniff se estaba quedando rezagada frente a otras aerolíneas en la carrera hacia la transición a una flota exclusivamente de jets. Desde el debut del Boeing 707 en 1959 hasta 1964, Braniff había logrado hacerse con diez unidades, suficientes para cubrir las rutas a Chicago, Nueva York y Washington. No obstante, el resto de sus rutas todavía operaban a partir de una flota de aviones con motor de pistón. Esta situación se hizo particularmente evidente en el caso de las rutas latinoamericanas, donde Braniff debía competir, hombro a hombro, con Pan Am y sus 707.

En marzo de 1965, Harding Lawrence, ejecutivo de la aerolínea, asume el cargo de director general y el reto de enfrentar, junto a su equipo, la transición total de la compañía a la era del jet. Fue así como Lawrence dio a conocer un nuevo plan de negocios que incluía la compra de docenas de aviones nuevos valorados en 160 millones de dólares, de los cuales la compañía tuvo que prestar 120 millones de dólares. Se sumaron catorce aviones nuevos (BAC-111) para la flota regional y diecisiete Boeing 727 nuevos para las rutas más largas, incluidas aquellas con destino a Latinoamérica. Lawrence también anunciaría que, para mediados de 1967, todos los aviones que no fueran de motor de reacción serían retirados de su flota (Braniff International Airways, 16 de junio de 1965). Para enero de 1966, la compañía había previsto ampliar su capacidad de asientos en vuelo en un 57\%, y para julio de 1966 , en un $27,5 \%$.

Lawrence enfrentaría, pues, el enorme desafío de incrementar la venta de boletos para hacer que esta inversión, y la deuda contraída, dieran resultados. Un elemento central en esta estrategia fue la nueva campaña publicitaria y de mercadeo para la cual Lawrence ya había identificado una aliada: Mary Wells, ejecutiva publicitaria de Jack Tinker and Partners, agencia con sede en la ciudad de Nueva York. En su autobiografía, Wells relata en detalle la urgente súplica de Lawrence: "Escúchame, Mary. Necesito una gran idea para esta aerolínea, algo tan grande que ponga a Braniff en primera plana de la noche a la mañana". Tras esa necesidad de atención estaba la compra de una nueva flota de aviones: "Voy a comprar una gran flota de aviones que costará mucho... No quiero poner a volar un montón de asientos vacíos". Lawrence reforzaría su petición sugiriendo un cambio de imagen total: "Quiero contratarlos a ustedes, a Tinker, para que me ayuden a relanzar Braniff en los Estados Unidos. En realidad, lo que quiero es presentar Braniff al mundo" (Wells Lawrence, 2002, p. 33). Luego, acordaría duplicar el presupuesto publicitario de la aerolínea, que alcanzaría los seis millones de dólares en el primer año (Braniff International Airways, 24 de noviembre de 1965).

Durante su investigación sobre el estado del transporte aéreo, Mary Wells dio forma a sus más audaces estrategias. A mediados de 1965, su equipo de publicistas pasó un tiempo en los aeropuertos de Estados Unidos, en los aviones y en las taquillas, donde entrevistaron a los viajeros aéreos. El hallazgo principal fue, a la vez, obvio y revelador: "Los aviones eran todos iguales; las terminales eran iguales; todas las azafatas eran iguales. Predominaba el gris, con dejos militares” (Wells Lawrence, citada en Black, 1975). Wells percibió, con razón, que muchos de los recursos de la aviación civil de los Estados Unidos estaban estrechamente vinculados a las movilizaciones militares de la Segunda Guerra Mundial y la Guerra Fría. En efecto, muchos aeródromos y terminales fueron construidos de forma apresurada a principios de la década de 1940; la mayoría de los pilotos fueron entrenados en la Segunda Guerra Mundial o en Corea; y las principales innovaciones tecnológicas -a saber, la propulsión a chorro, el sistema de presurización, cabinas más grandesfueron consecuencia de las investigaciones adelantadas por el ejército de los Estados Unidos durante la Segunda Guerra Mundial.

Sin embargo, los uniformes militarizados de las azafatas tienen su origen mucho antes, en la década de 1930. Sus tonos oscuros, las franjas en las mangas y las solapas decoradas con estilizadas alas se inspiran en la Marina de Estados Unidos, detalles que serían imitados por Pan Am -la primera gran aerolínea estadounidense-, en el uniforme de su tripulación a bordo de los "hidroaviones" que navegaban los cielos entre Miami y Sudamérica en esta primera década de la aviación civil. Por otro lado, los primeros viajes aéreos también fueron turbulentos, fríos y llenos de peligros potenciales para accidentes de aterrizaje; de ahí que, un uniforme monótono y pesado, que proyectara autoridad en tiempos de peligro, fuese apropiado (Tiemeyer, 2013). Al 
expresar su preocupación por aquel "dejo militar", Wells también podría haber querido señalar que su trabajo para Braniff estaba ocurriendo en paralelo a la movilización del ejército estadounidense en Vietnam. Cabe notar que los marines estadounidenses desembarcaron por primera vez en Danang en marzo de 1965, justo cuando Wells cierra su contrato con Braniff. Como tal, la decisión final de optar por tonos exuberantes, renunciar a las franjas en los puños y reemplazar las elegantes alas de la solapa por una paloma dorada de líneas acentuadas -símbolo de la paz- parece bastante intencionada. En consecuencia, cuando Wells y su equipo emprendieron la tarea de renovar la imagen de los vuelos en la era del jet, también buscaban evocar la paz en una industria estrechamente vinculada a la máquina de guerra de los Estados Unidos.

La inspiración inicial de la nueva campaña de Braniff fue el color, pues durante la investigación de su equipo, lo que a Wells le llamó más la atención fue la ausencia de este en los aeropuertos y aviones:

No había color. Ten en cuenta que eran los años sesenta, y el color era, por entonces, una herramienta de moda en el mercadeo... [A Harding Lawrence] le gustaba pensar en el color; me recordó que Braniff volaría a destinos, como México y Sudamérica, asociados con tonos vibrantes (Wells Lawrence, 2002, p. 34).

De hecho, el atractivo del turismo al sur de la frontera era precisamente escapar de la monotonía de los inviernos norteamericanos y de la estética monocromática de las rutinas laborales y escolares, aventurándose al sur, hacia las soleadas y coloridas tierras de la siesta obligada. El color tuvo, pues, mayor importancia en los Estados Unidos en la década de los sesenta, y también a nivel mundial, como tendencia generalizada en el diseño pensado para los consumidores de élite y la clase media. Como señala Shirley Kennedy, historiadora de la moda,

[Era] imposible no notar el color en todas partes. Los tonos vibraban y parecían estallar sobre las sedas diseñadas por [Emilio] Pucci, como en el arte pop de Lichtenstein, Warhol, Wesselman y Rosenquist,... en los carteles de los conciertos de rock psicodélico y en The Yellow Submarine, la película animada de los Beatles (Kennedy, 1991, p. 98).

Los tonos eran, pues, alegres, frescos y, como señaló Harding Lawrence, evocaban la supuesta simplicidad de las culturas precolombinas de América Latina. Al respecto, Mary Wells admitió: "El color fue idea mía, pero no realmente. No hay soluciones mágicas en la publicidad. Muchos no hacen su tarea, enfocándose en lo obvio" (Wells Lawrence, citada en Black, 1975). Lo necesario, desde su perspectiva, era combinar el jet ultramoderno con la magia esencial del color.

Finalmente, el color inundó todo lo que Braniff renovó bajo la dirección de Wells: mostradores de atención, salas de aeropuerto, uniformes de azafatas y materiales publicitarios. Pero la explosión inicial de este colorido cambio de imagen ocurrió en los propios aviones. Como señala Wells, primero consideró tener una flota toda en amarillo, en naranja o en índigo. Su director de arte hizo bocetos de los aviones en estos colores y luego los ubicó en el suelo para que el personal los criticara. "Luego le pedí que hiciera uno con todos los aviones de varios colores", recuerda Wells. "Cuando los bocetos estuvieron listos sobre el suelo de la recepción, fue una bomba. No había ninguna duda en mi mente... Siete colores parecían, wow, una gran idea, acogedores. Sería una gran noticia”. Enseñarle el concepto a Lawrence también fue fácil, un éxito sorprendente: "Cuando examinó los bocetos de sus aviones, con bloques de siete colores diferentes, se quedó callado durante un minuto. Creo que contuve la respiración. Luego, él se rió. Y dijo, nunca lo olvidaré, 'iLo lograremos!'” (Wells Lawrence, 2002, p. 35). Con el color como motivo principal, Mary Wells emprendería la tarea de abrir caminos y presentar a Braniff en la era del jet con una gran campaña que anunciaba "El fin del aeroplano plano". 


\section{EXOTISMO LATINOAMERICANO A LA VENTA: DEL “AEROPLANO PLANO" A LAS SOFISTICADAS PASARELAS AÉREAS}

Una vez aprobado el uso del color como estrategia principal para que Braniff se diferenciara en el mercado, Mary Wells tomó una segunda decisión vital, inclinándose por tendencia de acogida universal -el color-, estrechamente vinculada a impulsos más propios de la alta sociedad. Después de todo, Wells no empleó el color de forma ingenua, como podría encontrarse, por ejemplo, en una guardería infantil; tampoco optó por collages de colores propiamente "primitivos", como los que uno encontraría en los textiles precolombinos. Por el contrario, para la implementación del color en Braniff, Wells escogió a dos de los diseñadores más importantes de Estados Unidos y Europa, ambos célebres por su uso del color: del mundo de las pasarelas, a Emilio Pucci; y del diseño de interiores, a Alexander Girard. Al incorporar con éxito su estética en la aerolínea, Wells se aseguró de que la nueva paleta de Braniff tuviera toques elitistas.

Para Emilio Pucci, el célebre diseñador florentino, la adopción de una paleta psicodélica era de naturaleza dual: se prestaba bien a los fines de Braniff de vender el exotismo latinoamericano por vía de la tecnología ultramoderna del avión de reacción. Por un lado, los colores de Pucci y sus patrones geométricos simples surgieron de los viajes del diseñador por el sur global. Así, su interés por los textiles lo llevó a Bali, India, África meridional y México, encontrando en muchos de los textiles de estas sociedades premodernas un uso similar del color y una simplicidad que contrastaba drásticamente con la monotonía cromática occidental del siglo XX. Con pesar, Pucci se pronunciaría al respecto del declive en la variedad en la moda occidental, especialmente, para las mujeres en el mercado laboral, advirtiendo: "la mayoría de las azafatas van vestidas como si viajaran en autobús en 1925" (Pucci citado por Kennedy, 1991, p. 154). Sin embargo, lo que Braniff esperaba era que Pucci reemplazara aquellos "uniformes serios y masculinos" de las azafatas con toques femeninos de color en "faldas pantalón, leotardos, faldas cruzadas, bufandas, bombines y delantales de servicio" (Braniff International Airways, s.f.).

Sin embargo, el famoso diseñador se inspiró también, no en "lo primitivo", sino en lo ultramoderno. Antes de comenzar su carrera como diseñador de modas, Pucci había sido un apasionado de la aviación y se había formado como piloto de la fuerza aérea italiana durante la Segunda Guerra Mundial. Durante sus misiones de pilotaje, principalmente sobre los desiertos del norte de África, volaba a bajas altitudes para evitar los radares enemigos. Al recorrer las dunas del Sahara, iluminadas por los amaneceres o los atardeceres, y luego los pueblos y ciudades más vistosos a lo largo de la Costa Azul mediterránea, "Pucci evocaba el movimiento constante y el caleidoscopio de colores que se extendían ante él en estas largas misiones" (Kennedy, 1991, p. 9).

Por lo tanto, para el diseñador mismo hubo una fusión de lo primitivo y lo moderno en el uso del color, lo que permitió que sus diseños evocaran un carácter fantástico, muy contrario al legado de apacible inspiración victoriana de la Europa moderna. En la década de 1960, las mujeres lo suficientemente ricas como para comprar sus vestidos - como la famosa Sophia Loren o la glamorosa Primera Dama de los Estados Unidos, Jackie Kennedy- se sintieron atraídas por la vitalidad que irradiaban los trajes de noche de Pucci. Por su lado, sus diseños más atrevidos, con colores, igualmente llamativos, de telas ceñidas al busto, la cintura y las piernas, hicieron que las fanáticas de la moda más jóvenes y aventureras se sintieran desinhibidas y libres: "¿Cómo es que, en la década de los sesenta, las mujeres se atrevieron a recorrer las calles de la ciudad", se pregunta la teórica de la moda, Shirley Kennedy, "vistiendo prendas cortas, muy brillantes y sugestivas, ceñidas al cuerpo y con estampados salvajes?" (Kennedy, 1991, p. 9).

La exclusividad fue un ingrediente importante en el atractivo de Pucci. Sus vestidos eran populares no solo por ser explosivamente coloridos, sino también por las celebridades que los usaban. De hecho, Pucci comenzó su carrera atendiendo a un selecto círculo de clientes (por lo general, aristócratas de antaño) que compraban directamente en sus boutiques en zonas exclusivas de ciudades como Capri, en Italia. A mediados de la década de los sesenta, cuando el astuto hombre de negocios permitió una distribución más amplia de sus diseños, especialmente, en los grandes almacenes de los Estados Unidos, los nuevos ricos de Estados Unidos, cada vez 
más numerosos, fueron flechados por Pucci. Como señala Helen Gurley Brown, la exitosa autora y editora de Cosmopolitan, a pesar de la "democratización" de su cadena de suministro, los precios de Pucci seguían siendo prohibitivos:

Recuerdo ver mi primer vestido Pucci en Miami, en 1963, en los pasillos de Burdine, la tienda por departamentos, durante el evento promocional de un libro. “¿Cuánto tiempo llevamos en esto?", pensé, y aunque no creía que pudiera pagarme uno (190 dólares por un diminuto vestido), me probé cuatro en quince minutos por puro placer (Kennedy, 1991, p. 7).

El hecho de que Brown, autora de best sellers, se cuestionara si podía permitirse las creaciones de Pucci, habla de su exclusividad, incluso tras su llegada al gigante mercado de la moda en los Estados Unidos.

Ese mismo aire de exclusividad invadió el evento mediático que Braniff organizó para revelar los resultados de la propuesta de Pucci para la aerolínea: una sorprendente, colorida y vanguardista colección de uniformes para azafatas, que contrastaba drásticamente con los monótonos uniformes de inspiración militar de los competidores de la compañía. Fue así como, en julio de 1965, la historia de la aviación no despegaría desde una pista de hormigón, sino desde una pasarela en un lujoso salón de baile del Palazzo Pitti en Florencia, ciudad natal de Pucci. En el evento, en el que el diseñador presentaría su colección de alta costura para otoño, debutaría también su conjunto de uniformes de azafata. El resultado, en palabras de la revista Vogue, encarnaba la fluidez geográfica y la nostalgia extraterrestre de la era del jet: "En apariencia, mezcla de Texas, Florencia y Marte, la ropa que [Pucci] ha diseñado responde a cualquier necesidad posible, incluso, al futuro" (McCarty, 1965, p. 62). En sus comunicados oficiales, Braniff comentó algo similar:

Como piloto italiano condecorado, está abriendo un nuevo camino en la era espacial para la moda, hasta ahora terrestre. Su concepto para los atuendos de las azafatas de vuelo es absolutamente innovador, absolutamente contemporáneo y concuerda absolutamente con lo que él cree: "Cuando diseño, pienso en la mujer en movimiento" (Braniff International Airways, 19 de julio de 1965).

El diseñador rehuyó el término "uniforme" para sus uniformes de azafata multicolores, refiriéndose a ellos como una "colección de alta costura". La colección permitió a las azafatas lucir cuatro estilos distintos durante un mismo vuelo: una primera capa compuesta por un abrigo de invierno en un tono pastel brillante (amarillo, naranja o rojo), debajo del cual la azafata vestía un traje completo de falda cruzada y chaqueta con cremallera en otra paleta pastel completamente diferente (compuesta por tonalidades rosas, azules y lavandas). Debajo de esas prendas, las azafatas llevaban una capa más ligera, compuesta por una falda pantalón y una blusa cuello tortuga en otra combinación de colores pastel. El cuarto artículo, apodado el "Puccino", era una bata de dos colores que se usaba al servir la comida. Cada prenda simbolizaba esa combinación de elegancia, informalidad y sofisticación que hacía que los diseños de Pucci fueran tan deseados por las celebridades y las jóvenes de mentalidad independiente en los Estados Unidos: siluetas ceñidas al cuerpo y por encima de la rodilla que hacían atractivas a las mujeres en movimiento. Ya fuera en los anuncios de televisión o caminando por los aeropuertos de Estados Unidos y Latinoamérica, la impactante explosión de color de los atuendos diferenciaba a las azafatas de Braniff de sus competidores.

Con sus creaciones de varias capas, Pucci pretendía capturar la maravilla de los vuelos en jet. A su modo de ver, los jets de Braniff permitían saltar, de forma asombrosamente rápida, del clima helado de Chicago a las cálidas playas de Río de Janeiro. La azafata, atareada e intentando lucir glamorosa en medio de tan drásticos cambios de temperatura, se beneficiaría de las capas. Ahora, podría embarcar con un abrigo grueso, guantes, e incluso, un casco opcional -una burbuja de plástico inspirada en la era espacial y el traje astronauta- para cubrir su cabello de la lluvia o la nieve, y cambiar, así, poco a poco, su atuendo durante el vuelo. Unas horas después, al aterrizar - con un par de prendas menos-, luciría una falda pantalón y blusa más ligeras, lista para despedir a los pasajeros sobre el abrasador asfalto de una pista de aterrizaje en Río.

$\mathrm{Al}$ emplear telas ajustadas pero transpirables y fácilmente lavables, Pucci prestó especial atención a las exigencias propias del estilo de vida nómada de las azafatas. Y se sentía particularmente orgulloso de que todo el conjunto cupiera en un bolso de viaje. "En el futuro", señaló, "todo lo que un viajero internacional 
podría necesitar añadir a ese conjunto sería un vestido o dos y algunos accesorios para la noche" (Braniff International Airways, julio de 1965). Como tal, incluso en esa especie de alegre y primitiva interpretación del color, Pucci otorgaba movilidad a las mujeres modernas de la era del jet, lo que para él era vital: "El movimiento es muy importante en nuestras vidas", decía. "Una mujer en falda corta puede correr para tomar un taxi y lucir elegante, pero si corre en falda larga, puede lucir torpe" (Kennedy, 1991, p. 139).

De ahí que, refiriéndose al diseñador florentino, Marilyn Bender explicara su relevancia para el mundo de la moda en la década de los sesenta, centrándose en la tecnología del jet. Al señalar que los inicios de los sesenta marcaban "el umbral de la era del jet", la periodista y escritora insistió en que "los vestidos de Pucci eran símbolo y pasaporte de una nueva era. De aspecto frágil pero indestructibles, elegantes y sexis, eran el armario-móvil en cápsula para la mujer activa que se precia de un cuerpo hermoso" (Kennedy, 1991, p. 46). No debe pasarse por alto el hecho de que en 1964 había trece millones de mujeres solteras en Estados Unidos y otros 23 millones de mujeres casadas trabajando fuera del hogar (Scanlon, 2010, p. 144). Los viajes en avión, inevitablemente, aumentarían, incluso para las mujeres trabajadoras sin compañía masculina. Por lo tanto, con su "colección de alta costura" para las azafatas, Pucci hacía un esfuerzo por ayudar a las mujeres trabajadoras a verse divertidas e impredecibles, destacando su nueva movilidad.

Entretanto, el diseñador de interiores Alexander Girard fue otro miembro de la élite del Atlántico Norte cuya pasión por el color se originó también en el sur global. Aunque nació en Nueva York, se crió en Italia y se educó en Inglaterra, fue en Latinoamérica y las zonas fronterizas de Estados Unidos donde Girard más se sintió como en casa. Mantuvo su residencia principal en Santa Fe, Nuevo México, y algunos de sus primeros trabajos con textiles lo llevaron al centro de México, donde trabajó en una fábrica textil del siglo XIX, aun cuando su clientela se encontraba, principalmente, en metrópolis como Nueva York y San Francisco. La asignación de tareas que Mary Wells estableció para Pucci y Girard, sus dos maestros diseñadores, fue bastante simple: Pucci diseñaría los trajes de las azafatas y, dado su gusto por captar la atención de los medios, sería él quien acudiría a los eventos de prensa; por su lado, Girard rediseñaría todo lo demás para la aerolínea. Esto incluyó un nuevo logotipo, una terminal de facturación de colores brillantes en el aeropuerto de origen de Braniff en Dallas y una serie completa de espacios deslumbrantes para que los pasajeros pudieran relajarse: interiores lujosos y de colores llamativos en los aviones, sofisticados clubes de primera clase y una atractiva puerta en las salas de embarque. Para Mary Wells, nada debía ser ignorado en el encuentro visual de los clientes con la marca, y sería Girard, más que Pucci, quien se ocuparía de esto.

Dos aspectos influyeron en la decisión de Mary Wells de trabajar con Girard: la pasión del diseñador por el arte popular latinoamericano, que impregnaría gran parte de lo que diseñó para Braniff, además de su inclinación por los colores vibrantes y su capacidad para incorporar formas de inspiración "premoderna" en los espacios arquitectónicos que, en la década de los sesenta, definirían "lo moderno". El ejemplo más famoso sería la audaz propuesta de Girard para La Fonda del Sol, el restaurante en Midtown Manhattan, que fusionaba la arquitectura brutalista del Time \& Life Building cerca del Rockefeller Center con la calidez de colores vibrantes y el arte popular mexicano. Al igual que en el caso de Pucci y sus prendas de lujo, el trabajo en diseño de interiores de Girard solo estaba al alcance de una selecta clientela: los altos ejecutivos que visitaban las sedes de sus clientes -como, por ejemplo, las elegantes oficinas de John Deere en Illinoiso los comensales asiduos de la ya mencionada y excesivamente costosa Fonda del Sol. De hecho, Mary Wells conocía a Girard porque a ella y a sus hijas -quienes vivían cómodamente de su salario de seis cifras- les encantaba frecuentar el restaurante, al cual consideraban "una potente mezcla de colores, un encuentro entre lo mexicano y lo moderno" (Wells Lawrence, 2002, p. 35). Otra apreciación semejante acerca del trabajo de Girard en la Fonda del Sol, aunque más depurada, apareció en la revista Interiors, la cual afirmaba que "la simpleza de su propuesta la hacía aún más encantadora" (The Inn of the Sun, 1961, p. 89).

Para completar su propuesta, Girard también le prestó a la compañía numerosas piezas de su extensa colección privada de arte popular latinoamericano. De ahí que, en los interiores de sus aviones, Braniff exhibiera cerámica azteca y textiles incas, al igual que tallados mayas en las paredes frontales las cabinas de clase 
turista. Lo mismo ocurriría en el salón de primera clase del aeropuerto Love Field, en Dallas, donde la división de ambientes se hizo a partir de vitrinas que exhibían piezas en cerámica fina, entre otras, pertenecientes a civilizaciones de la América precolombina. El efecto final que se quería lograr con Braniff, tanto como con la Fonda del Sol en Nueva York, era una mezcla inspiradora entre lo ultramoderno (los viajes aéreos y el avión de reacción) y lo premoderno (arte popular primitivo). Dicho asincronismo resultaría una analogía válida para la experiencia que Braniff deseaba promocionar entre los turistas estadounidenses con destino a América Latina: la oportunidad de volar en la forma de transporte aéreo más avanzada disponible, con el objetivo de regresar a una suerte de estado humano primigenio de simplicidad, de encanto, de esparcimiento, de ingenua inocencia ante un nuevo descubrimiento. ${ }^{4}$

\section{CONCLUSIONES: UN CÓCTEL EN LAS NUBES}

La propuesta estética de "modernidad premoderna" desarrollada para Braniff por el trío compuesto por Mary Wells, Emilio Pucci y Alexander Girard fue clave en el cumplimiento del objetivo que se había propuesto Lawrence, su director general: llenar los asientos en los nuevos aviones de Braniff. Así, para junio de 1966, el tráfico de pasajeros de la aerolínea había aumentado en un 48,7\% con respecto al año anterior (Newman, 1966). Durante el primer año de la campaña, sus ingresos aumentaron de manera similar en un 42\% (Loomis, 1968, p. 114). Esto se debió a dos logros simultáneos en la captación de nuevos clientes. Para los clientes de primera clase, compuestos, principalmente, por ejecutivos de empresas y sus esposas, la acogida del brillo y la exclusividad -y el glamour de Pucci y Girard-, eran razones suficientes para ser seducidos y abandonar Pan Am. Pero serían muchos más los clientes de clase turista -de ingresos y poder adquisitivo considerablemente inferiores- que volarían por primera vez con Braniff en los sesenta. Para ellos, la renovada estética de Braniff también sería muy atractiva. Por entonces, el controlado mercado de la aviación, estrictamente regulado por entes locales y por la Asociación Internacional de Transporte Aéreo (IATA), vigilaba que todas las aerolíneas ofrecieran sus vuelos internacionales a la misma tarifa. Las únicas diferencias eran el tipo de aviones empleados, -de ahí el interés de Braniff por actualizar su flota y alcanzar a Pan Am, poniendo más aviones en servicio- y, por supuesto, la calidad del servicio.

Los agentes publicitarios que, a finales de los sesenta, trabajaban para Pan Am e investigaban los hábitos de los viajeros con presupuestos limitados, encontraron lo siguiente: “Al [comprometerse a] pagar la tarifa plena, estos clientes están dispuestos a elegir una aerolínea entre la oferta centrándose en aspectos relacionados con la comodidad, el servicio y la satisfacción" (J. Walter Thompson Company, 1969, p. 18). Con sus nuevos jets y su elegante transformación, Braniff parecía ofrecer más a estos clientes por su dinero. Al pagar las elevadas tarifas por sus traslados aéreos, estos clientes accedieron a atenciones más propias del jet set: pudieron relejarse en las salas de espera diseñadas por Girard, embarcar en aviones con texturas audaces e, incluso, observar obras de arte latinoamericano cuidadosamente seleccionadas por el mismo Girard; además, fueron atendidos por azafatas que lucían el mismo tipo de atuendos que llevaban las estrellas de cine vestidas por Pucci. En términos generales, estos clientes de clase media y sus ocasionales acompañantes -pertenecientes a la clase trabajadora y ahora viajeros en clase turista - tuvieron la oportunidad de colarse y hacer parte de la atmósfera y los ambientes propios de las películas y las celebridades. Ya fuera joven o mayor, hombre o mujer, soltero o casado, rico o de clase media, cualquier cliente podía acceder así a la anhelada estética del universo Braniff. En este sentido, se podría decir que, gracias a sus colaboraciones con los diseñadores de élite Emilio Pucci y Alexander Girard, la compañía había logrado su objetivo de democratizar la era del jet.

La decisión de Braniff de convertir a sus azafatas en anfitrionas vestidas de Pucci también desviaba la atención de la creciente crisis de personal de tripulación, ligada a la democratización de la era del jet. Ahora, con aviones más grandes y rápidos, los clientes temían que los viajes aéreos se parecieran más al transporte público masivo: marcados por el utilitarismo en vez de la exclusividad, congestionados e impersonales, en lugar de agradables. En el lenguaje de la campaña publicitaria de Mary Wells se advertía un profundo 
temor a los viajes aéreos en "aeroplanos planos". Este hallazgo está respaldado por investigaciones posteriores realizadas en 1969, momento en que Pan Am estaba a punto de estrenar el jumbo jet 747. Las encuestas realizadas entre los consumidores de ingresos medios revelaron su gran preocupación por el hecho de que los aviones se volvieran demasiado grandes: "Su impresión también pone de relieve la preocupación de fondo por la deshumanización del viaje aéreo. La percepción es que el pasajero será un individuo más en una multitud y que no recibirá el tipo de atención personalizada que busca" (J. Walter Thompson Company, 1969, p. 16). Ciertamente, los aviones jumbo, con capacidad potencial para más de 400 asientos, podrían originar tal percepción. Sin embargo, ya con la primera generación de aviones de reacción, los modelos 707 y 727 que Harding Lawrence compró para Braniff en 1965, surgían dudas. El DC-7C de 1955, el modelo de avión previo a la era del jet más espacioso que Braniff había tenido, contaba con 75 asientos, mientras que los nuevos 727 tenían capacidad para 154 pasajeros. ${ }^{5}$ Los viajeros de clase turista que buscaban un trato personalizado a bordo del avión se decepcionarían con mayor frecuencia.

En consecuencia, las azafatas se enfrentaban, pues, a clientes con expectativas inalcanzables. Como señala un informe de Pan Am, "Los consumidores... desean ser tratados como individuos. Algunos de ellos son incluso sensibles a la simpatía ejemplar de las azafatas. No quieren un saludo mecánico por agradable que sea. Quieren sentir que alguien realmente se preocupa por tratarlos como individuos" (J. Walter Thompson Company, 1969, p. 18). Sin embargo, brindar este tipo de atención individualizada en la era del jet sería cada vez más difícil para las auxiliares de vuelo, ya que tendrían que atender a más pasajeros y seguir llevando a cabo tareas complejas como preparar y servir platos estupendos y ofrecer bebidas frecuentemente en vuelos de menor duración. Para hacer frente a expectativas tan poco realistas, los trajes de azafata diseñados por Pucci para Braniff ofrecían una posible solución. Al convertir los pasillos de sus aviones en pasarelas de moda, se estaría invitando a los pasajeros a verse más como espectadores que invitados de un cóctel. De esa forma, continuarían siendo parte del sofisticado mundo del jet set al que aspiraban, pero de forma pasiva, lo que estaría más en sintonía con el estilo impersonal de los viajes de esta era.

En definitiva, la decisión de Mary Wells de "acabar con el aeroplano plano" a través de esta fórmula sirve de lección para las relaciones públicas no solo del sector de la aviación en la década de 1960, sino para los efectos visuales que, incluso hoy, los destinos turísticos latinoamericanos se ven obligados a crear -también, ilusiones epistemológicas- para seguir seduciendo a los turistas estadounidenses con destinos turísticos como paisajes soleados de ensueño, sencillez y encanto. En 1965, Wells y su equipo creativo aceptaron la nueva realidad de que el transporte aéreo en sí mismo ya no era maravilloso. Hubo, pues, un tiempo en que la tecnología de la aviación era tan asombrosa que los clientes abordaban con una sensación de asombro. También hubo una época en la que el solo encanto de los remotos destinos ofrecidos por la aerolínea se vendía sin mayores esfuerzos. Pero el equipo de Braniff tendría que aceptar que esas nociones heredadas ya no tenían validez, al menos no la suficiente como para llenar todos los asientos extra que ofrecía un jet.

La situación que Braniff enfrentaba en 1965 era la misma que preocupaba a todas las aerolíneas durante la transición al jet: la duplicación de la capacidad de asientos y una enorme deuda, lo que demandaría la democratización de su producto. Para Braniff, esto implicaría volver su experiencia de vuelo llamativa, convirtiéndola en todo un evento. Las propuestas de Pucci y Girard propiciarían un ambiente dinámico -en especial, a partir de la explosión de color-, gracias a la mezcla de sofisticación y alegría que lo acompañaba. Todo esto se haría evidente en los rediseños de Girard para la terminal y en la nueva vibrante paleta de la compañía. También se hizo visible en los audaces, juveniles y elegantes uniformes diseñados para las azafatas por Pucci. Como señalaría Lawrence, Braniff tenía como objetivo acabar con la "monotonía" en la experiencia del viajero frecuente y "ofrecer a todos nuevas razones para volar" (The Return of Braniff, in Technicolor, 1966, p. 23). 


\section{FUENTES DE ARCHIVOS DOCUMENTALES CONSULTADAS}

Archivo "Alexander Girard”, Museo de Diseño Vitra (Vitra Design Museum), Weil am Rhein, Alemania: Braniff International Airways. (16 de junio de 1965). Sin titulo [comunicado de prensa], caja "MAR 00110 D-5", carpeta "Girard Press - 01 / 1966-12/1966".

Braniff International Airways. (19 de julio de 1965). Emilio Pucci: Fashion Innovator" [comunicado de prensa], caja "MAR 00105 D-4", carpeta "Girard Press - 01/1964 - 12/1965".

Braniff International Airways. (julio de 1965). World Fashion Press Acclaims Pucci-BraniffFlight Fashions, en The Braniff B-Liner, Caja "MAR 00105 D-4", Carpeta "Girard Press - 01/1964 - 12/1965".

Colección Braniff en la Colección History of Aviation Collection, Universidad de Texas en Dallas, Richardson, Texas, Estados Unidos:

Braniff International Airways. (24 de noviembre de 1965). New Look [memorando], caja 34, carpeta 2.

Braniff International Airways. (s.f.). A Braniff International hostess is [folleto].

"Harding Lawrence Markets an Airline". (julio de 1968). Media Decisions, caja 18, carpeta 4.

Archivo "Emilio Pucci", Palazzo Pucci, Florencia, Italia.

Colecciones de la agencia J. Walter Thompson Company. Biblioteca de libros raros y manuscritos de David M. Rubenstein, Duke University, Durham, Carolina del Norte, Estados Unidos:

J. Walter Thompson Company. (1969). Preliminary Exploration of Consumer Perceptions of the 747 Plane in England, France and Germany [informe de mercado para Pan American World Airways Inc.], Colecciones de la agencia J. Walter Thompson Company.

\section{REFERENCIAS BIBLIOGRÁFICAS}

Black, C. (25 de marzo de 1975). Meet America's Top Woman Exec. Honolulu Advertiser.

Cass, R. B. (2015). Braniff Airways: Flying Colors. Mt. Pleasant, Carolina del Sur: Arcadia Publishing.

Kennedy, S. (1991). Pucci: A Renaissance in Fashion. Nueva York: Abbeville Press.

Loomis, C. (1968). As the World Turns-On Madison Avenue. Fortune (diciembre).

McCarty, J. (1965). Beauty Checkout. Vogue, 146 (5).

Newman, C. (8 de junio de 1966). Color It Colorless: Black and White Gain in Fashions and Homes. Wall Street Journal.

The Inn of the Sun (1961). Interiors, 120 (7), 88-99.

The Return of Braniff, in Technicolor. Air Travel (enero de 1966).

Scanlon, J. (2010). Bad Girls Go Everywhere: The Life of Helen Gurley Brown. Nueva York: Penguin.

Sinatra, F. (1958). Come Fly With Me. Billy May (compositor), Jimmy Van Heusen y Sammy Cahn (letristas). Los Ángeles: Capitol Records.

Stadiem, W. (2014). Jet Set: The People, the Planes, the Glamour, and the Romance in Aviation's Glory Year. Nueva York: Ballantine Books.

Tiemeyer, P. (2013). Plane Queer: Labor, Sexuality, and AIDS in the History of Male Flight Attendants. Berkeley: University of California Press.

Wells Lawrence, M. (2002). A Big Life in Advertising. Nueva York: Knopf.

\section{Notas}

1 Este texto fue entregado originalmente en inglés y traducido por Estefanía Calderón Potes. La traductora señala la doble acepción de la palabra inglesa "runway", que significa por un lado "pista de aviación" y, por otro, "pasarela de moda". En el contexto de este ensayo, una coincidencia semántica fantástica. Otra anotación: el modo de citación en este texto 
en algunos casos difiere de la citación APA utilizada en el resto del dosier. La citación de fuentes de archivos históricos necesita más precisión; esta fue aplicada a petición expresa del autor.

2 La traductora ha optado por emplear la voz inglesa jet, o avión de reacción.

3 En 1952, los jets Comet de la British Overseas Airways Corporation (BOAC) hicieron su debut. Sin embargo, solo serían puestos a tierra a partir de 1954 debido a una serie de accidentes que requirieron importantes modificaciones en su diseño.

4 En 1978, Girard donó su extensa colección de arte popular contemporáneo (la mayoría proveniente del actual territorio mexicano) al Museo Internacional de Arte Popular en Santa Fe, donde todavía está disponible para el público. Ver: ht tp://www.internationalfolkart.org/about/ourhistory/girardwing.html

5 Los aviones previos a la era del jet más grandes que Braniff tendría son los DC-7C, estrenados a partir de 1955. El avión tenía capacidad para un máximo de 81 pasajeros, pero las entregados a Braniff eran para 62 pasajeros (todos en primera clase) o para 75 pasajeros (distribuidos entre primera clase y clase turista). Por su parte, los 707 originales de la aerolínea contaban con 106 asientos, con casi la misma cantidad de primera clase y de clase turista, si bien un 707 podía alcanzar hasta 210 pasajeros. Los 727 de Braniff a veces volaban con una capacidad de 154 pasajeros de clase turista, si bien Boeing los comercializaba con capacidad para hasta 189 pasajeros. 